\section{Observations beyond the limit}

\section{Demosthenes Kazanas}

Cygnus $\mathrm{X}-3$ is one of the brightest and most extraordinary objects in our Galaxy. It was first discovered as an X-ray source in 1966 and identified as the source of a giant radio outburst in 1972 . Subsequent observations of Cyg X-3 have heralded the extension of the energy range for astronomy first to teraelectron volts $(1 \mathrm{TeV}=$ $\left.10^{12} \mathrm{eV}\right)$ and then to petaelectron volts (1 $\left.\mathrm{PeV}=10^{35} \mathrm{eV}\right)$. Now Cassiday et al. ', using the 'Fly's Eye' detector in Utah, have observed Cyg X-3 at higher energies still - exaelectron volts $\left(1 \mathrm{EeV}=10^{18} \mathrm{eV}\right)$ and above. The authors estimate the probability that the excess of cosmic rays at this energy observed from the direction of Cyg X-3 could occur by chance in a uniform distribution of showers is only $6 \times 10^{-4}$

Collisions of high-energy primary cosmic rays in the upper atmosphere initiate showers of secondary particles which can be observed by astronomers. The Fly's Eye detector used by Cassiday et al. maps the nitrogen fluorescence excited in the atmosphere by such showers and has a coverage of $2 \pi$ steradians. Since November 1986, the operation of two such detectors has permitted three-dimensional reconstructions of the showers. The $\mathrm{TeV}$ cosmic rays are observed via the Cerenkov (electromagnetic shock wave) radiation emitted by charged secondaries. And PeV rays initiate showers of electrons (and muons) observed directly by detectors on the ground. Each technique has a resolution of $1^{\circ}$, so that point sources can be identified.

\section{Interest and scepticism}

Since its introduction in the early 1980 s the field of astronomy at energies above $1 \mathrm{TeV}$ has been viewed with both interest and scepticism? ${ }^{2}$. The interest stems from the apparently counterintuitive fact that a large fraction (a tenth) of the luminosity of galactic accreting objects is emitted at energies above $1 \mathrm{TeV}$ - well above the potential energy at the surface of even a neutron star - and in some cases up to $1,000-10,000 \mathrm{TeV}$. The scepticism arises from the rather low statistical significance of the observations owing to the small number of particles arriving from these sources at these energies: at such high energies, only a few particles are needed to contribute significantly to the total luminosity of an object. It was hoped that new larger detectors would settle the difficulties with statistical significance.

Unfortunately, observations of Cyg X-3 with one of these detectors reported recently ${ }^{3}$ provide only upper limits to the flux at PeV energies, and the observers arrived at the unsatisfactory conclusion that PeV emission ought to be episodic. This conclusion is nonetheless strengthened by observations of another source, Her X-1, an X-ray pulsar (period 1.2378 s), which has also been detected in the $\mathrm{TeV}$ and PeV range ${ }^{+6}$. These observations indicate that at energies of $1 \mathrm{TeV}$ or more, the signal from this source appears in bursts lasting anything from a few minutes to a hundred. A search for periodicities in these bursts indicates a period of $1.2357 \mathrm{~s}-$ an intriguing result, as this is significantly different from the period of the X-rays. To compound the puzzles, the muon content in the PeV showers is inconsistent with the assumption that they are initiated by photons (if they were, there should have been ten times fewer muons).

Instead, the muon content is consistent with that expected from hadron-initiated showers. But any such particles, if electrically charged, would be disturbed while traversing the turbulent interstellar magnetic field, so that the observed directional and temporal coherence of the showers could not have been preserved. And there are no known stable neutral particles that could fit the description.

Amid all these puzzles and problems there has been a significant recent advance which may put the whole field on a much firmer ground ${ }^{7}$ : the detection of a statistically highly significant (nine-standarddeviation), constant signal of $1-\mathrm{TeV}$ photons from the Crab nebula (background showers initiated by cosmic-ray hadrons were identified and subtracted). This is by far the most significant veryhigh-energy detection and was achieved by imaging the showers' Cerenkov radiation, a technique which allows the rejection of 98 per cent of the background events. The importance of this result lies in that it provides a weak $\left(10^{34} \mathrm{erg} \mathrm{s}^{-1}\right)$ but steady 'standard candle' against which future experiments will be calibrated.

What can be learned from all these observations? First, a large fraction of the accretion energy in many $\mathrm{X}$-ray sources is converted (albeit sporadically) into relativistic protons with energies greater than $1 \mathrm{TeV}$ (it is generally agreed that it is too difficult to accelerate electrons to these energies) which subsequently produce the observed high-energy radiation through nuclear collisions. Several models for these sources have been proposed (see ref. 8 for a review) which, however, are rather unconstrained owing to the scarcity of photons and lack of repeatability of the observations. Their most important feature is the estimate of the maximum energy expected (about $10^{16}-10^{17} \mathrm{eV}$ ) by the acceleration mechanism favoured by each model (shock acceleration near the compact object or large-scale static electric fields).

Given their simplicity, the agreement of these models with the observations in the $10^{15}-10^{16}-\mathrm{eV}$ range is rather remarkable. With the maximum particle energy already set by the models, it would seem that the new observations of Cassiday et al. ${ }^{\prime}$ are in direct conflict with theory, especially if the observed particles are photons, which would have to be generated at the source by particles of energy $10^{19} \mathrm{eV}$. Cassiday et al. point out, however, that the observed showers could be due to neutrons. (The Fly's Eye cannot distinguish between photon- and neutron-induced showers; also neutrons, although unstable, can get to Earth at these energies without much attenuation from decay.) Indeed, Ellison and I have proposed ${ }^{9}$ that neutrons are copiously emitted from these objects and could be observed on Earth.

\section{High-energy tail}

Furthermore, if the spectrum of the protons accelerated in the source decreases exponentially above the energy of maximum intensity suggested by the current models $\left(10^{17} \mathrm{eV}\right)$, the energy flux at $1 \mathrm{EeV}$ should be $\exp (10) \approx 10^{4}$ times smaller than that at lower energies, in rough agreement with the observations obtained with the Fly's Eye. These observations might therefore probe the high-energy end of the particle distribution that produces the radiation at $\mathrm{PeV}$ energies.

As for the muon content of the showers. the situation remains confused. An obvious solution is an unknown strongly interacting particle; more appealing, to my taste, is the recent proposal ${ }^{\text {II }}$ that the quantum-chromodynamic structure of the photon causes an increase in the photoproduction cross-section for particle collisions at energies of $0.5 \mathrm{TeV}$ in the centre of mass. At these energies, the photons can interact with nuclei in the atmosphere by the nuclear strong force, a process which may compete with the electromagnetic ones considered so far in the shower development and enhance the muon content. These showers might hence be pointing to new physics at these energies, to be investigated with further observations and detailed modelling.

Demosthenes Kazanas is at the NASA Goddard Space Flight Center, Greenbeit, Maryland 20771, USA.

\footnotetext{
1. Cassiday. G.L. et al. Phys. Rev. Lett. 62, 383-386 (1989).

2. Protheroe, R.J. Proc. 20th int. Conf. Cosmic Rays 8. 21 (1987).

3. Dingus, B.L. et al. Phys. Rev. Lett. 60, 1785-1788 (1988)

4. Lamb, R.C. et al. Astrophys. J. 328, L13-L16 (1988)

5. Resvanis, L.K. et al. Astrophys. J. 328, L9-L12 (1988).

6. Dingus. B.L. et al. Phys. Rev. Lett. 61, 1906-1909 (1989).

7. Weeks. T.C. et al. Astrophys. J. (in the press)

8. Hillas, A.M. Proc. 19th int. Conf. Cosmic Rays 9. 407 (1985).

9. Kazanas, D. \& Ellison, D.C. Nature 319. 380 (1986).

10. Drees, M. \& Halzen. F. Phys. Rev. Lett. 61, 275 (1988).
} 